Research Article

\title{
Comparative Transcriptome Analysis in Eggplant Reveals Selection Trends during Eggplant Domestication
}

\author{
Qingzhen Wei, ${ }^{1}$ Liming Du, ${ }^{1}$ Wuhong Wang, ${ }^{1}$ Tianhua Hu, ${ }^{1}$ Haijiao Hu, ${ }^{1}$ Jinglei Wang, \\ Karine David $\mathbb{C}^{2},{ }^{2}$ and Chonglai Bao $\mathbb{D}^{1}$ \\ ${ }^{1}$ Institute of Vegetable Research, Zhejiang Academy of Agricultural Sciences, Hangzhou 30021, China \\ ${ }^{2}$ The University of Auckland, School of Biological Sciences, Private Bag 91019, Auckland 1010, New Zealand \\ Correspondence should be addressed to Karine David; k.david@auckland.ac.nz and Chonglai Bao; baocl@mail.zaas.ac.cn
}

Received 7 February 2019; Accepted 15 April 2019; Published 9 May 2019

Academic Editor: Hieronim Jakubowski

Copyright (C) 2019 Qingzhen Wei et al. This is an open access article distributed under the Creative Commons Attribution License, which permits unrestricted use, distribution, and reproduction in any medium, provided the original work is properly cited.

\begin{abstract}
Eggplant (Solanum melongena L.) is an economically and nutritionally important fruit crop of the Solanaceae family, which was domesticated in India and southern China. However, the genome regions subjected to selective sweeps in eggplant remain unknown. In the present study, we performed comparative transcriptome analysis of cultivated and wild eggplant species with emphasis on the selection pattern during domestication. In total, 44,073 (S. sisymbriifolium) to 58,677 (S. melongena cultivar S58) unigenes were generated for the six eggplant accessions with total lengths of $36.6-46 \mathrm{Mb}$. The orthologous genes were assessed using the ratio of nonsynonymous $\left(K_{\mathrm{a}}\right)$ to synonymous $\left(K_{\mathrm{s}}\right)$ nucleotide substitutions to characterize selective patterns during eggplant domestication. We identified 19 genes under positive selection across the phylogeny that were classified into four groups. The gene (OG12205) under positive selection was possibly associated with fruit-related traits in eggplant, which may have resulted from human manipulation. Eight positive selected genes were potentially involved in stress tolerance or disease resistance, suggesting that environmental changes and biotic stresses were important selective pressures in eggplant domestication. Taken together, our results shed light on the effects of artificial and natural selection on the transcriptomes of eggplant and its wild relatives. Identification of the selected genes will facilitate the understanding of genetic architecture of domesticated-related traits and provide resources for resistant breeding in eggplant.
\end{abstract}

\section{Introduction}

Over human history, wild plants and animals were selected and adapted for cultivation and commercial use, a process known as domestication. Darwin proposed that domestication and adaptation were in parallel in the wild and are powerful in generating phenotypic diversity [1]. Selection in the wild and during domestication follows similar processes, which involves the selection of beneficial alleles present in the wild germplasm or arose via spontaneous mutations at a collection of loci controlling yield and quality. Studies on several organisms suggest that very few genetic loci contributed to the rapid phenotypic divergence relative to domestication [2-6].

With the improvements of sequencing technologies and increasing availability of plant genomes, the consequences of domestication could be studied at a transcriptional or even whole-genome level. Several domestication-associated genes were characterized in some crops such as rice, maize, tomato, and pepper [7-10]. A recent study in maize showed that 46 genes were putative targets of selection with functions mostly related to biotic stress [9]. In tomato, around 50 genes were under positive selection during domestication, the adaptation to extreme environments caused a broad alteration of transcriptional networks, and the sequences of genes were involved in environmental and stress responses [8]. Despite the importance of the absolute changes in gene expression or changes in regulation networks during domestication, the selective patterns among domesticated species and multiple wild relatives are still limited to a few organisms.

Brinjal eggplant (Solanum melongena L., $2 n=2 x=24$ ) is an economically important vegetable crop and is widely grown in America, Europe, and Asia, with 51.3 million tons of agricultural production in 2016 (http://faostat.fao.org). 
The eggplant belongs to the large family Solanaceae, which compromises $>3000$ species with diverse genetic and phenotypic variation including tomato, pepper, and potato. Eggplants exhibit a wide biodiversity of local landraces/wild relatives with huge variation respect to growth habit, pathogen resistance, and fruit size and color. The subgenus Leptostemonum ("spiny solanums") comprises approximately 500 species, including three closely related cultivated eggplant species: S. melongena (brinjal eggplant, section Melongena), S. macrocarpon (gboma eggplant, section Melongena), and S. aethiopicum (scarlet eggplant, section Oliganthes), which are all of Old World origin and grown as food crops [11]. The domestication region of eggplant has long been debated; two most commonly hypothesized regions are India and southern China/Southeast (SE) Asia [12], where eggplant use has equally old written records dating approximately 2000 years ago. Both of the hypothesized regions have highly diverse landraces and populations of candidate progenitors of domesticated eggplants (S. incanum L. in India and $S$. undatum L. in southern China). Domesticated eggplant exhibits substantial phenotypic differences from its wild progenitors, reflecting rapid and pronounced evolutionary changes. Identification of genetic changes underlying these phenotypic differences will give insight into the genetic architecture of complex traits, the response to selection, and provide resources for eggplant improvement.

The availability of the eggplant genome (S. melongena $\mathrm{cv}$. Nakate-Shinkuro) [13] provides an excellent opportunity for performing comparative analysis at the transcriptome level. In recent years, various studies have been performed on comparative transcriptomics to investigate molecular mechanisms underlying disease-resistant, fruit-related traits and develop molecular markers in eggplant [14-17]. However, the knowledge of changes in gene expression or transcriptional regulation networks during domestication is still lacking. In the present study, we investigated gene divergence accompanied with eggplant domestication by comparative transcriptomics between wild and domesticated eggplants. We examined the transcriptomes of three cultivated eggplant accessions (S58, HZHQ, and LYQ) and three wild relatives (S. aethiopicum, S. integrifolium, and S. sisymbrifolium). Evidence for significant changes in both gene expression levels and sequences was found which might have occurred as a result of selective sweeps and specifically identified a subset of genes that were likely targets of selection during domestication.

\section{Materials and Methods}

2.1. Plant Materials, Growth, and RNA Isolation. Six different eggplants were used in the present study, three wild species (S. sisymbriifolium, S. aethiopicum, and S. integrifolium) and three domesticated eggplant cultivars from $S$. melongena (S58, HZHQ, and LYQ) (Figure 1). Two eggplant cultivars, HZHQ and LYQ, are widely grown across China (HZHQ in Zhejiang and LYQ in Beijing); both are purple blackskinned and have either a long fruit or an oval fruit (Figure 1). The seeds of HZHQ and LYQ were collected by the Institute of Vegetable Sciences (Zhejiang Academy of

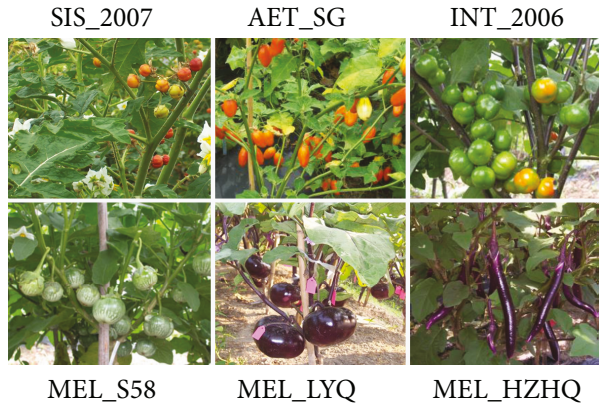

FIgUre 1: The fruit and leaf morphology of eggplant (Solanum melongena) and wild relatives. AET_SG: S. aethiopicum L.; SIS_2007: S. sisymbriifolium L.; INT_2006: S. integrifolium; MEL_S58, MEL_HZHQ; MEL_LYQ: S. melongena.

Agricultural Sciences), whereas the seeds of eggplant cultivar S58 were provided by the Asian Vegetable Research and Development Center (http://www.avrdc.org, Thailand branch), and it is cultivated in Thailand. The seeds of $S$. sisymbriifolium, S. aethiopicum, and S. integrifolium were obtained from Xishuangbanna Botanical Garden (Yunnan, China). S. aethiopicum is mostly grown in tropical Africa [18]; however, S. aethiopicum has four classifications (Gilo, Shum, Kumba, and Aculeatum); S. integrifolium (S. aethiopicum gr. Aculeatum) is one of them and mainly used as rootstocks (mansfeld.ipk-gatersleben.de). S. aethiopicum and $S$. integrifolium belong to the section Oliganthes which forms a sister group to $S$. melongena within the Old World clade [12], whereas S. sisymbriifolium belongs to the Sisymbriifolium clade, distant from the Old World clade [19].

Seeds (10 per species) from the six eggplants were germinated in soil and in the dark for 7 days. Plants were then transferred into growth chambers $\left(28^{\circ} \mathrm{C} / 22^{\circ} \mathrm{C}\right.$ day/night temperatures and $14 \mathrm{~h}$ photoperiod with $300 \mu \mathrm{mol}$ photons $\left.\mathrm{m}^{-2} \mathrm{~s}^{-1}\right)$. When the plantlets had three true leaves, the leaf tissue was collected from the seedlings 14 days postgermination. The leaf tissue for each species was collected from three individual plants then mixed for RNA extraction. Total RNA of each sample was extracted using an RNAprep Pure Plant Kit (DP432, TIANGEN, China, http://www.tiangene.com) according to the manufacturer's protocol.

2.2. cDNA Library Construction, Sequencing, and Assembly. Illumina sequencing was performed at Novogene Bioinformatics Technology Co. Ltd., Beijing, China (http:// www.novogene.cn). mRNA was purified from $3 \mu \mathrm{g}$ total RNA using poly-T oligo-attached magnetic beads. Sequencing libraries were generated using Illumina TruSeq ${ }^{\mathrm{TM}} \mathrm{RNA}$ Sample Preparation Kit (Illumina, San Diego, USA) following the manufacturer's recommendations. In order to select cDNA fragments of preferentially $200 \mathrm{bp}$ in length, the library fragments were purified with the AMPure XP system (Beckman Coulter, Beverly, USA), then sequenced on an Illumina Hiseq 2000 platform, and 90 bp paired-end reads were generated. Clean reads were obtained by removing reads containing adapter and poly- $\mathrm{N}$ and low-quality reads from raw data. All the downstream analyses were based on 
clean data with high quality. Transcriptome assembly was accomplished based on the left files and right files using Trinity [20] with min_kmer_cov set to 2 by default and all other parameters set by default.

2.3. Annotation, GO, and KEGG Pathway Enrichment Analysis. To annotate the unigenes of the eggplant transcriptomes, each of the unigenes from all six eggplants was searched against different public databases, including KEGG Orthology database, Gene Ontology (GO) database, NCBI nonredundant protein sequences (NR) database, protein family (PFAM) database, Swiss-Prot protein database, NCBI nucleotide sequence (NT) database, and Eukaryotic Orthologous Group (KOG) database. GO enrichment analysis of genes was implemented by the GOseq $\mathrm{R}$ package-based Wallenius noncentral hypergeometric distribution [21], which can adjust for gene length bias. KEGG [22] is a database resource for understanding high-level functions and utilities of the biological system, such as cell, organism, and ecosystem, from molecular-level information, especially large-scale molecular datasets generated by genome sequencing and other high-throughput experimental technologies (http://www.genome.jp/kegg/). We used KOBAS [23] software to test the statistical enrichment of differential expression genes in KEGG pathways.

2.4. Alignment of RNA-seq Data to the Reference Eggplant Genome. Reference genome and gene model annotation files were directly downloaded from the eggplant genome database (http://eggplant.kazusa.or.jp/). The index of the reference genome was built using Bowtie v2.0.6, and pairedend clean reads were aligned to the reference genome using TopHat v2.0.9. We selected TopHat as the mapping tool for TopHat can generate a database of splice junctions based on the gene model annotation file and thus a better mapping result than other nonsplice mapping tools.

The Cufflinks v2.1.1 Reference Annotation-Based Transcript (RABT) assembly method was used to construct and identify both known and novel transcripts from TopHat alignment results. Picard-tools v1.96 and SAMtools v0.1.18 were used to sort, mark duplicated reads, and reorder the bam alignment results of each sample.

2.5. Quantification of Gene Expression Levels. Gene expression levels were estimated by RSEM [24] for each sample. Clean data were mapped back onto the assembled transcriptome, and HTSeq v0.5.3 (EMBL, Heidelberg, Germany) was used to count the read numbers mapped to each gene. RPKM (reads per kilobase of exon model per million mapped reads) considers the effect of sequencing depth and gene length for the read count at the same time and is currently the most commonly used method for estimating gene expression level [25].

2.6. Validation of RNA-seq Data by Real-Time Quantitative PCR. RNA-seq data for 16 different genes was validated by real-time quantitative PCR (qPCR). The primers of selected genes were designed using Primer Priemer 5 software (PREMIER Biosoft, Palo Alto, CA, USA) and synthesized by Sangon. The primer pair SmActin-F and SmActin-R was used to amplify an eggplant actin fragment as a control for normalizing the starting amounts of cDNA. The sequences of primers used in this study are listed in Supplementary Table S4. The first-strand cDNA was synthesized using $1 \mu \mathrm{g}$ total RNA by HiScript II Q Select RT SuperMix for qPCR (+gDNA wiper) (Vazyme, Nanjing, China) in $10 \mu \mathrm{L}$ of reaction mixture. The quantitative PCRs were performed according to the manufacturer's instructions using the DyNAmo ${ }^{\text {TM }}$ Flash SYBR Green qPCR Kit (Tiangen Biotech, Beijing, China) in an ABI Prism StepOnePlus real-time thermal cycler (Applied Biosystems, Carlsbad, CA, USA). The amplification was performed as follows: $10 \mathrm{~min}$ at $94^{\circ} \mathrm{C}$ followed by 40 cycles of $94^{\circ} \mathrm{C}$ for $20 \mathrm{~s}$ and $60^{\circ} \mathrm{C}$ for $60 \mathrm{~s}$. A melting curve was generated to ensure product uniformity. Gene expression was evaluated by the $2^{-{ }^{\triangle} \triangle} \mathrm{Ct}$ method [26]. The expression of genes was related to the SmActin expression. The correlation between expression profiles of selected genes obtained from real-time PCR and RNA-seq data based on $\log 2$ RPKM values was determined using MS Excel 3.7.

2.7. $K_{\mathrm{a}} / K_{\mathrm{s}}$ Analysis among Domesticated and Wild Eggplants. In genetic analysis, the $K_{\mathrm{a}} / K_{\mathrm{s}}$ ratio represents the ratio of the number of nonsynonymous substitutions per nonsynonymous site $\left(K_{\mathrm{a}}\right)$ to the number of synonymous substitutions per synonymous site $\left(K_{s}\right)$, which could be used as an indicator of selective pressure acting on protein-coding genes. In order to estimate the $K_{\mathrm{a}} / K_{\mathrm{s}}$ ratio, the bidirectional best hit $(\mathrm{BBH})$ method [27] was used to search the orthologous genes among 6 eggplants. $K_{\mathrm{a}} / K_{\mathrm{s}}$ calculation was performed with the PAML package using default settings [28]. Comparisons of homologous genes with a high $K_{\mathrm{a}} / K_{\mathrm{s}}$ ratio significantly $>1$ are usually considered to be evolving under positive selection, whereas $K_{\mathrm{a}} / K_{\mathrm{s}}$ ratio $<1$ indicates purifying selection on the gene loci, and $K_{\mathrm{a}} / K_{\mathrm{s}}$ ratio close to 1 indicates neutral mutation.

\section{Results and Discussion}

3.1. De Novo Transcriptome Sequencing and Assembly of Different Eggplants. Molecular analyses have been conducted to evaluate genetic diversity among wild and domesticated eggplant species using various approaches such as RAPD, SSR, and sequence analysis [29-32]. However, the changes in transcriptional networks and sequence divergence that accompany with domestication remain unknown. To assess selection pressure and the extent of transcriptome variation that occurred during $S$. melongena domestication, the leaf transcriptomes of three $S$. melongena cultivars, HZHQ (MEL_HZHQ), LYQ (MEL_LYQ), and S58 (MEL_S58), and distant relatives, S. aethiopicum (AET_SG), S. integrifolium (INT_2006), and S. sisymbriifolium (SIS_2007), were analyzed.

RNA samples from leaf tissues of each species were prepared for Illumina sequencing to generate the transcriptome data of eggplant. A total of more than 426 million reads were generated (ranging from 55 to 72 million reads for each sample), with 381 million Illumina reads (89.45\%) passing the quality filtering. The average Phred quality score was at 
TABLE 1: Information of RNA-seq data for the six eggplants.

\begin{tabular}{|c|c|c|c|c|c|c|}
\hline \multirow{2}{*}{ Sample } & \multirow{2}{*}{ Raw reads } & \multirow{2}{*}{ Clean reads } & \multirow{2}{*}{ GC (\%) } & \multicolumn{2}{|c|}{ Phred quality Q20 (\%) } & \multirow{2}{*}{ Total mapped ${ }^{\mathrm{a}}$} \\
\hline & & & & -1 & -2 & \\
\hline AET_SG & 61795572 & 55580924 & 41.99 & 97.32 & 95.85 & $39337841(70.78 \%)$ \\
\hline INT_2006 & 74782564 & 67387058 & 42.51 & 97.21 & 96.03 & $48949822(72.64 \%)$ \\
\hline MEL_HZHQ & 65869048 & 58960600 & 41.68 & 97.28 & 96.09 & $51659443(87.62 \%)$ \\
\hline MEL_LYQ & 66197610 & 59365428 & 42.35 & 97.27 & 95.81 & $52647559(88.68 \%)$ \\
\hline MEL_S58 & 75562708 & 67428684 & 41.68 & 97.3 & 95.93 & $58745699(87.12 \%)$ \\
\hline SIS_2007 & 82147678 & 72679508 & 42.45 & 97.18 & 96.10 & $20027221(27.56 \%)$ \\
\hline Total & 426355180 & 381402202 & & & & \\
\hline
\end{tabular}

${ }^{a}$ The number of high-quality reads mapped using TopHat and the ratio with the clean reads.

TABLE 2: Summary feature of the eggplant transcriptome assemblies.

\begin{tabular}{|c|c|c|c|c|c|c|c|c|}
\hline \multirow{2}{*}{ Sample } & \multicolumn{4}{|c|}{ Transcript } & \multicolumn{4}{|c|}{ Unigene } \\
\hline & Number & Total length $(\mathrm{Mb})$ & Average length (bp) & N50 (bp) & Number & Total length $(\mathrm{Mb})$ & Average length (bp) & N50 (bp) \\
\hline AET_SG & 100296 & 13.3 & 1322 & 2186 & 51519 & 43.8 & 850 & 1576 \\
\hline INT_2006 & 82045 & 10.1 & 1239 & 2025 & 45838 & 40.5 & 463 & 1606 \\
\hline MEL_HZHQ & 88368 & 98.4 & 1113 & 1876 & 55118 & 43.1 & 420 & 1368 \\
\hline MEL_LYQ & 98897 & 12.4 & 1257 & 2081 & 52819 & 44.8 & 430 & 1568 \\
\hline MEL_S58 & 101387 & 12.1 & 1193 & 2010 & 58677 & 46 & 408 & 1408 \\
\hline SIS_2007 & 73161 & 77.1 & 1053 & 1716 & 44073 & 36.6 & 450 & 1469 \\
\hline
\end{tabular}

TABLe 3: The number and ratio of the CDS, annotated genes, and mapped sequences for the six eggplants.

\begin{tabular}{|c|c|c|c|c|c|c|c|}
\hline \multirow{2}{*}{ Sample } & \multirow{2}{*}{$5^{\prime} \mathrm{UTR}^{\mathrm{A}}$} & \multirow{2}{*}{$3^{\prime} \mathrm{UTR}^{\mathrm{A}}$} & \multicolumn{2}{|c|}{ CDS } & \multirow{2}{*}{ Total CDS ratio(\%) ${ }^{\mathrm{B}}$} & \multirow{2}{*}{$\begin{array}{l}\text { Number of unigenes } \\
\text { annotated }^{\mathrm{C}}\end{array}$} & \multirow{2}{*}{ Total mapped ${ }^{\mathrm{D}}$} \\
\hline & & & Blast & EST Scan & & & \\
\hline AET_SG & $12123(23.53 \%)$ & $11626(22.57 \%)$ & 21195 & 18652 & 77.34 & $29118(56.51 \%)$ & $39337841(70.78 \%)$ \\
\hline INT_2006 & $12830(27.99 \%)$ & $12393(27.04 \%)$ & 20952 & 15564 & 79.66 & $28321(61.78 \%)$ & $48949822(72.64 \%)$ \\
\hline MEL_HZHQ & $14230(25.82 \%)$ & $13570(24.62 \%)$ & 26867 & 18952 & 83.13 & 34865 (63.25\%) & $51659443(87.62 \%)$ \\
\hline MEL_LYQ & $12908(24.44 \%)$ & 12405 (23.49\%) & 21749 & 19162 & 77.45 & $29751(56.32 \%)$ & $52647559(88.68 \%)$ \\
\hline MEL_S58 & $13867(23.63 \%)$ & $12992(22.14 \%)$ & 26486 & 20652 & 80.33 & 35659 (60.77\%) & $58745699(87.12 \%)$ \\
\hline SIS_2007 & $13523(30.68 \%)$ & $14051(31.88 \%)$ & 24478 & 11525 & 81.69 & $29221(66.3 \%)$ & $20027221(27.56 \%)$ \\
\hline
\end{tabular}

${ }^{\mathrm{A}}$ The number of $5^{\prime}$ UTR or $3^{\prime}$ UTR and the ratio with the unigenes. ${ }^{\mathrm{B}}$ The total CDS ratio with the unigenes. ${ }^{\mathrm{C}}$ The number of unigenes annotated to the database and the ratio with the all unigenes. ${ }^{\mathrm{D}}$ The number of high-quality reads mapped to the reference eggplant genome and the ratio with the clean reads.

least $95 \%$ for $99 \%$ base accuracy for filtered reads, indicating the high quality of filtered reads (Table 1). The Trinity software (trinityrnaseq_r2012-10-05) was used for de novo assembly of high-quality clean read sequences into transcript reads, ranging from 73,161 transcripts for S. sisymbriifolium to 101,387 for $S$. melongena S58. Only the longest transcripts were defined as final unigenes, yielding 44,073 (S. sisymbriifolium) to 58,677 (S. melongena S58) unigenes of at least $200 \mathrm{bp}$ in length for all species, with an average length of 408-850 bp and a total length of 36.6-46 Mb (Table 2). N50 being a weighted median statistic such that $50 \%$ of the entire assembly is contained in transcripts equal to or larger than this value in bp; The N50 value of the unigenes was between $1,368 \mathrm{bp}$ and 1,606 bp (Table 2). The decrease in N50 values between transcripts and unigenes suggests that longer genes tend to generate more isoforms [33]. Overall, the average number of unigenes for the three $S$. melongena accessions was 55,500. Across the six selected eggplants, the N50 values were between 1,368 bp and 1,576 bp, which are higher than those in Yang et al.'s study [33].

3.2. CDS Prediction, Functional Annotation, and Sequence Comparison. Genome annotation was then performed to provide important and pertinent information on gene function and structure. The coding sequence (CDS) of all unigenes was predicted by searching against the NCBI nonredundant (NR) and Swiss-Prot protein databases using ESTScan (3.0.3) or BLAST. The upstream and downstream sequences of the CDS in each unigene were considered as the $5^{\prime}$ and $3^{\prime}$ UTR sequences. $22 \%-31 \%$ unigenes contained the $5^{\prime}$ or $3^{\prime}$ UTR sequence, and $77 \%-83 \%$ CDS sequence among all sample was found from unigenes (Table 3). S58 contained the most unigenes among all the species analyzed, whereas S. sisymbriifolium had the highest ratio of UTR and 
CDS per unigene (Table 3). We annotated between $56.32 \%$ (MEL-LYQ) and 66.3\% (SIS-2007) unigenes using an $E$ -value threshold of $1 e-5$. The number of annotated genes for S. Melongena is comparable to the study published by Yang et al. [33], indicating high quality of the assembly presented in this study. The lack of annotation for some transcripts is due to the number of small transcripts generated in de novo assembly that did not show significant similarity with known proteins in various databases.

Clean reads for all samples were aligned to the eggplant reference genome (S. melongena var. Nakate-Shinkuro) using the TopHat software $[13,34]$. The three S. melongena cultivars mapped $\sim 88 \%$ of the published eggplant genome reads, whereas S. aethiopicum and S. sisymbriifolium mapped $71 \%$ and $27 \%$ respectively (Table 3 ). The mapping results also identified novel transcripts for the six eggplants that are not present in the current release of the eggplant reference genome (Figure 2). As expected, S. sisymbriifolium contained the most count of the novel transcripts (1257 genes), followed by $S$. aethiopicum (579 genes), S. integrifolium (459 genes), and S. melongena cultivars (323 genes for S58, 302 genes for HZHQ, and 244 genes for LYQ).

\subsection{Characterization of Sequence Diversity among Different} Eggplants. The genus Solanum contains up to 1400 species, the two most well-studied crop species tomato and potato are both from the New World, whereas the eggplant is of Old World origin and belongs to the subgenus Leptostemonum [12, 33]. The Old World subgenus Leptostemonum includes S. melongena (section Melongena), S. macrocarpon L. (section Melongena), and S. aethiopicum L. (section Oliganthes). The origin of the eggplant lineage is most likely started in Africa; however, the relationships among African species and their Asian relatives are not well understood [12]. To further investigate the phylogenetic relationship between species within the Old World clade and that from a more distant clade, we characterized the amount and nature of the genes that are either common or specific to $S$. melongena (HZHQ), S. aethiopicum, S. integrifolium, and S. sisymbriifolium. The published reference eggplant genome (S. melongena Nakate-Shinkuro) was also included in the analysis. Orthologous genes among the six species were determined using predicted protein sequences and the OrthoMCL algorithm (OrthoMCL Software-v2.0.3) [16, 35]. Genes from different species were considered as orthologues if the shared homology in their deduced amino acid sequences (BlastP, $e<0.00001$ ) was more than $80 \%$ of the size of the genes being compared. Overall, 2,695 singlecopy genes and 1,793 multicopy genes were common to all five species analyzed (Figure 3(a)). Interestingly, the two $S$. melongena cultivars, Nakate-Shinkuro and HZHQ, had the highest number of specific genes (5,835 and 3,692 respectively, Figure $3(\mathrm{a})$ ) with a ratio of specific to total genes being around 30\% (Figure 3(b)). On the other hand, $S$. aethiopicum and S. sisymbriifolium had a lower number of species-specific genes with 1,713 and 657 genes, respectively (Figure 3(a)); the number of specific genes for S. integrifolium was 939 genes. The results indicate that S. melongena, which has been subjected to intense domestication, has

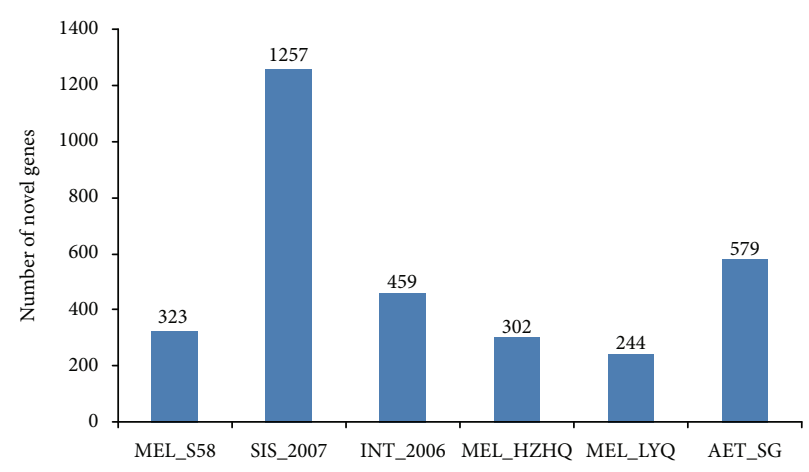

Figure 2: Number of novel transcripts. Histogram of the genes that are not mapped to the current release version of Nakate-Shinkuro reference genome in each of the analyzed samples.

seen its pool of new gene sequences increased compared to other relatives. Moreover, the results also support the fact that $S$. aethiopicum has not been subjected to such an intense domestication.

Based on sequence similarity with the genes of other species, the specific genes for the five eggplant species presented in Figure 3(a) were assigned a Gene Ontology (GO) function. Several GO items were not found in wild species especially S. sisymbriifolium, such as locomotion in biological process and items within a cellular component, which contain more specific genes and specific GO items (Supplementary Tables S1 and S2). Compared to the distant wild relatives S. aethiopicum and S. sisymbriifolium, cultivated eggplants have a number of genes with novel GO items such as locomotion, positive regulation of biological process, cell junction, extracellular matrix part, receptor activity, and synapse, which were not found in $S$. sisymbriifolium (Supplementary Table S1). However, the two GO items cell killing and channel regulator activity were lost in cultivar species HZHQ. The data shows that during domestication, eggplant has undergone the evolvement of a more complex molecular and metabolic function. Furthermore, the cultivated eggplant species has experienced rapid and strong adaptation in response to the fluctuating environmental conditions, but at the same time, some functions were lost.

3.4. Phylogenetic Relationships among the Wild and Cultivated Eggplants. Bayesian inference methods were then used to construct a phylogenetic tree using the single-copy orthologous gene sequences with the reference genomes of eggplant (Nakate-Shinkuro, MEL_NASH) [13], potato (Solanum tuberosum) [36], tomato (Solanum lycopersicum) [37], and pepper (Capsicum annuum) [10]. The resulting phylogenetic tree (Figure 3(c)) shows that the two domesticated and semiwild cultivars were belonging to the same clade; MEL_HZHQ and S58 were in a sister clade. Two of the wild species, $S$. aethiopicum and S. integrifolium, are in a sister clade; this is somehow supporting the classification that $S$. integrifolium is one of the four groups in S. aethiopicum, whereas the wild species $S$. sisymbriifolium was most distant from the domesticated species. This category result is consistent with previous reports $[12,19]$. Moreover, the 


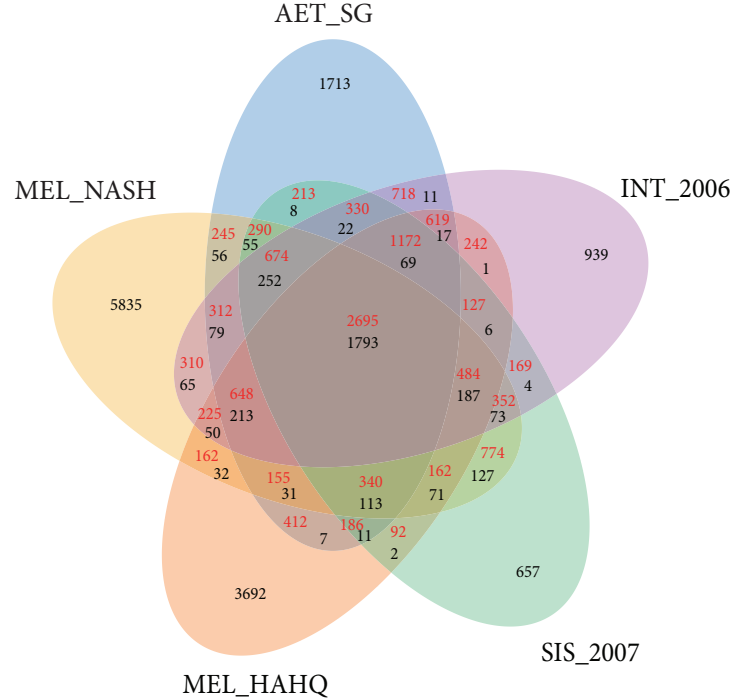

(a)

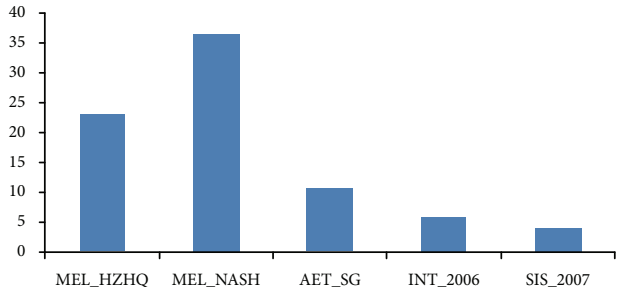

(b)

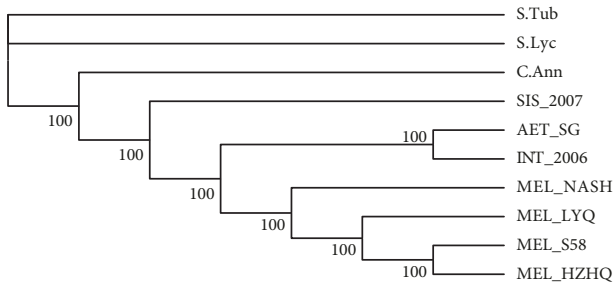

(c)

FIGURE 3: (a) Venn diagram exhibiting the comparison of orthologous gene sequences among the wild Solanum species S. sisymbriifolium (SIS_2007), S. aethiopicum (AET_SG), S. integrifolium (INT_2006), and S. melongena cultivar HZHQ (MEL_HZHQ) and NakateShinkuro (MEL_NASH) transcriptome. The number of common or specific genes is list in the circle. The shared genes among five species are categorized as single copy (1:1, red letters) and multicopy ( $\mathrm{N}$ : N; black letters). (b) The histogram showing the ratio of species-specific genes. (c) The phylogenetic tree constructed using OrthoMCL based on the sequences of orthologous genes in the six eggplant samples and other Solanaceae family species (C. annuum, S. lycopersicum, and S. tuberosum). All of the nodes have 100\% bootstrap support values.

cultivated pepper was found more closely related to eggplant than cultivated potato and tomato (Figure 3(c)). However, Wang et al. [38] and Song et al. [18] showed that eggplant was most closely related to potato and tomato clade than pepper. This difference in topologies is possibly resulting from incomplete gene sorting in eggplant leaf transcriptome.

\subsection{The Expression Divergence among Different Eggplants.} We further examined the differential expression in leaf tissues of the six eggplant accessions and detected the expression of orthologous genes. Interspecific comparisons based on pairwise gene expression differences revealed a striking decrease in the S. sisymbriifolium (SIS_2007) gene expression branch length (Figure 4(a)). Hierarchical clustering of the expression profiles in leaf tissues of all species showed that the two cultivated eggplants HZHQ and S58 were included into the same sister cluster, whereas two wild relatives $S$. aethiopicum (AET_SG) and S. integrifolium (INT_2006) were in the same cluster (Figure 4(b)). The results revealed that species within the same clade has similar gene expression pattern.

We compared gene expression data to identify genes showing evidence of differential expression among different eggplants. Wild relatives of cultivated crops usually possess allele resistance to pathogens, diseases, and extreme environment stresses. For example, S. aethiopicum provides source of abiotic and biotic resistance for cultivated S. melongena, and S. sisymbriifolium is the source of bioactive compounds [39-43]. In the present study, the number of genes with expression changes and KEGG pathway was significantly increased in all comparisons with the wild S. sisymbrififolium than any other lineages (Table 4). KEGG pathway enrichment analysis of these genes revealed overrepresentation of identified genes involved in stress response, metabolism, defense response, photosynthesis, response to pathogen, and redox pathways (Supplementary Table S3). Moreover, we identified genes involved in plant-pathogen interaction and plant hormone signal transduction pathway in the upregulation comparisons between other eggplants and S. sisymbriifolium (highlighted in red; Supplementary Table S3). Therefore, we propose that the transcriptional scope of S. sisymbriifolium is highly diverged from other five eggplants and that environmental stress has played a major role in driving transcriptional variations among different eggplants.

To validate the RNA-seq data, qPCR of 16 randomly selected genes was performed. As shown in Figure 4(c), there was a strong positive correlation $\left(R^{2}=0.81\right)$ between RNAseq data and qPCR data. The qPCR expression quantities were basically consistent with their transcript abundance changes identified by RNA-seq, which confirms the reliability of RNA-seq data.

3.6. Analysis of Positive Selection in the Wild and Cultivated Eggplant. The genetic basis of domestication-related traits has been studied in several organisms, including important crops such as maize, millet, rice, and cucumber [4, 44-46]. It is proposed that the rapid phenotypic divergence associated with domestication is often controlled by a limited number of genetic loci [6]. In maize, population genetic analysis and whole-genome resequencing have revealed that a small proportion of genes/genome region ( $5 \%)$ show evidence of positive selection during domestication [44, 47]. To assess selection pressure and the extent of transcriptome variation 
Cluster analysis of differentially expressed genes

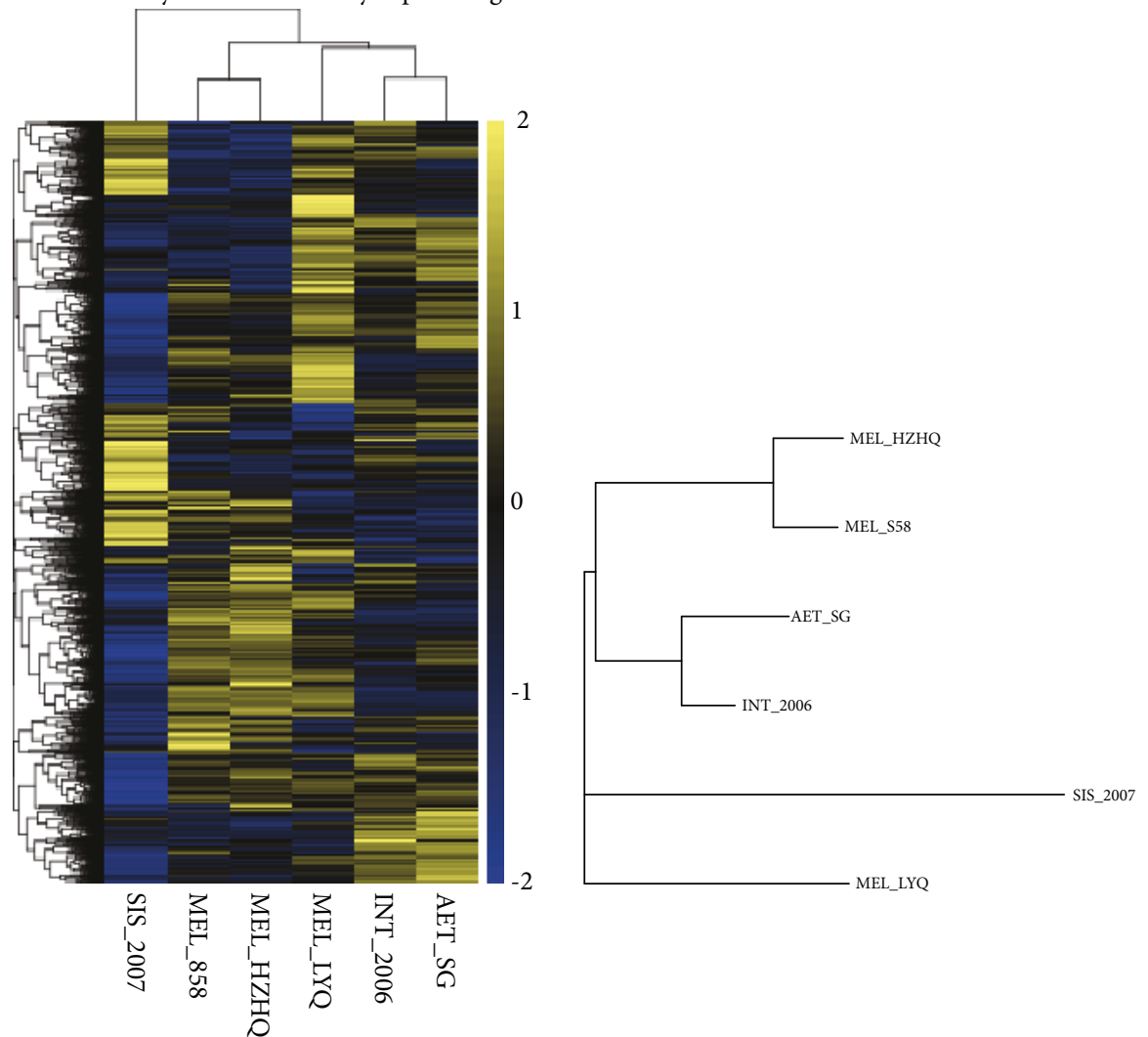

(a)

(b)

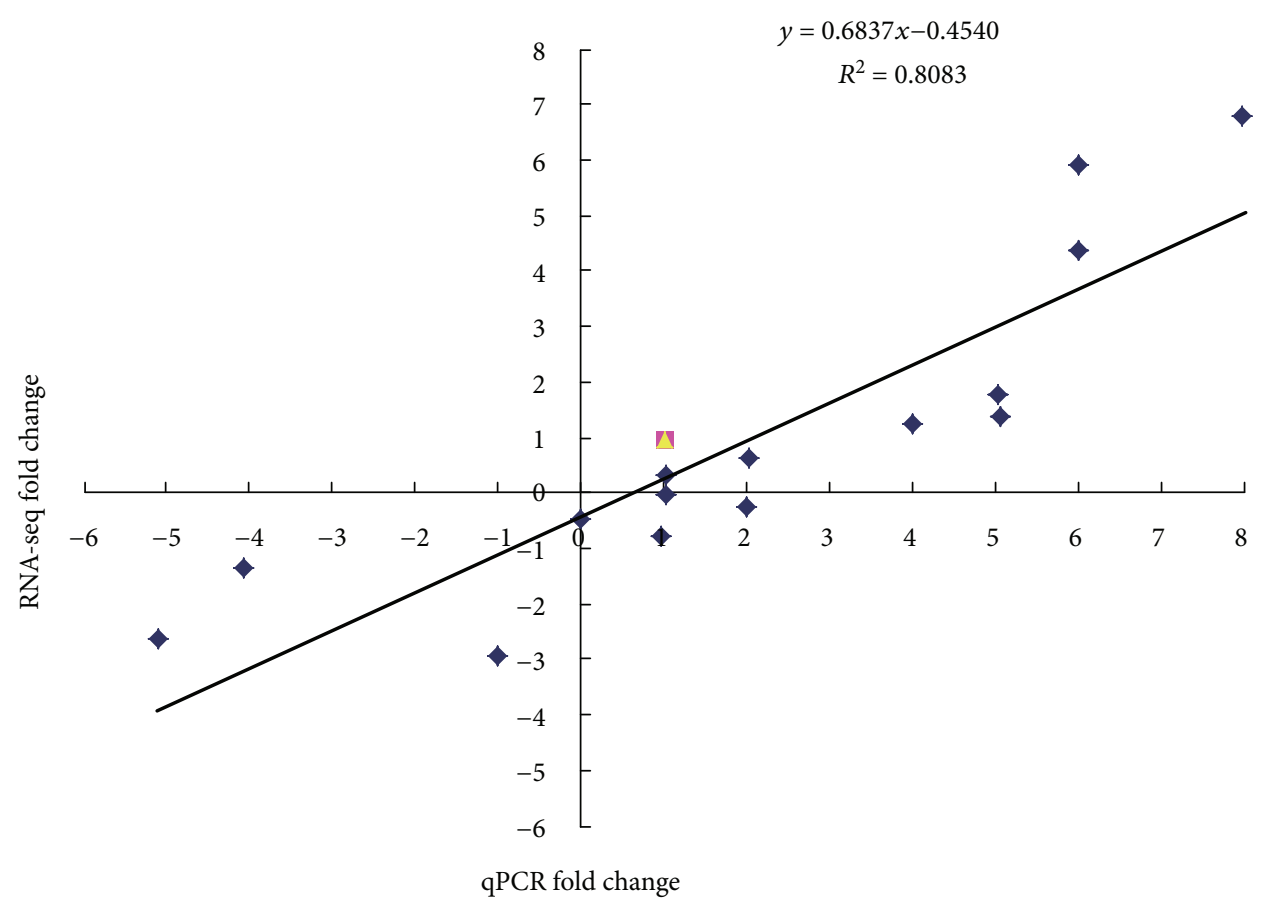

(c)

Figure 4: (a) The heatmap depicting expression profiles of orthologous genes in the six eggplant species S. sisymbriifolium (SIS_2007), S. aethiopicum (AET_SG), S. integrifolium (INT_2006), and S. melongena cultivars HZHQ (MEL_HZHQ), LYQ (MEL_LYQ), and S58 (MEL_S58). Scaled log2 expression values are shown with yellow and blue indicating high and low expressions, respectively. (b) Hierarchical clustering of the differentially expressed genes using the RNA-seq data derived from six samples based on RPKM values. (c) Correlation of fold change values from RNA-seq and qPCR based on the SmActin expression. The $R^{2}$ value is 0.808 . 
TABLE 4: The number of genes with expression changes in interspecific comparisons.

\begin{tabular}{lc}
\hline Comparison sample name & DEG $^{\text {a }}$ number \\
\hline MEL_HZHQ-AET_SG & 3578 \\
MEL_HZHQ-INT_2006 & 2955 \\
MEL_HZHQ-SIS_2007 & 5834 \\
MEL_LYQ-AET_SG & 3007 \\
MEL_LYQ-INT_2006 & 3110 \\
MEL_LYQ-SIS_2007 & 6473 \\
MEL_S58-INT_2006 & 2734 \\
MEL_S58-SIS_2007 & 5678 \\
SIS_2007-AET_SG & 5591 \\
SIS_2007-INT_2006 & 4302 \\
\hline
\end{tabular}

${ }^{a}$ DEG: differentially expressed genes.

which occurred during $S$. melongena domestication, the leaf transcriptome of the six eggplant accessions was analyzed. The ratio of $K_{\mathrm{a}} / K_{\mathrm{s}}$ by random substitution throughout the coding gene within specific loci can be used to estimate selective pressure; a gene with $K_{\mathrm{a}} / K_{\mathrm{s}}>1$ indicates that this gene is subjected to positive selection, whereas $K_{\mathrm{a}} / K_{\mathrm{s}}<1$ indicates purifying selection, and $K_{\mathrm{a}} / K_{\mathrm{s}}$ score close to 1 indicates neutral mutation [48]. From comparison of gene-level estimates of $K_{\mathrm{a}} / K_{\mathrm{s}}$ in all species, we identified 19 unigenes (listed in Table 5) that exhibited statistically significant $(P<0.05)$ evidence of evolution under positive selection across the phylogeny. Many of these genes have been annotated in tomato and potato and characterized in details in rice and Arabidopsis. The result supports the hypothesis that relatively few mutations have gone through strong selective sweeps during domestication.

The 19 genes subjected to positive selection could be divided into four groups: hormone response, development and response to disease and abiotic stress, oxidationreduction pathway, and development. Orthologous genes OG06215, OG08277, and OG12205 are likely related to the hormone response. OG06215 is characterized as a Domon superfamily which is auxin-induced protein in root cultures. OG08277 is a ribosomal protein, with annotated functions of controlling vacuole trafficking and developmental programs through the regulation of lipid metabolism in Arabidopsis. Ribosome proteins serve as translational regulators of auxin response, and lipid metabolism modulates auxin-mediated tissue differentiation [49]. Auxin plays a major role in the dynamic and complex phytohormone regulatory networks controlling fruit development [50]. In the present study, OG12205 was identified as an Aux/IAA family gene; its orthologous genes in tomato interact with ARF (auxin response factor) proteins and involved in the regulation of quality parameters over tomato fruit development and play diverse roles in flower and fruit development [51-53].

Many gene/protein families are reported to be associated with stress tolerance and disease resistance, such as PPR (pentatricopeptide repeat) protein, WRKY, E3 ubiquitin ligase, and DnaJ homolog subfamily proteins [54-59]. Park et al. [60] found that cleavage of PPR protein mRNAs renders
Arabidopsis thaliana more susceptible to pathogenic bacteria and fungi. PPR proteins also involved in chloroplast, mitochondrial and seed development, and abiotic stress response in rice and maize $[5,53,54,61]$. DnaJ homolog subfamily proteins as cochaperones also have critical functions in biotic and abiotic stress response. Overexpression of tomato chloroplast-targeted DnaJ protein enhances tolerance to drought stress and resistance to Pseudomonas solanacearum in transgenic tobacco [62]. We identified eight genes with annotated functions of response to disease and abiotic stress in other organisms (Table 5). For example, the gene $O G 0372$ functions as pentatricopeptide repeatcontaining protein; one gene (OG10870) belongs to the DnaJ homolog subfamily. A series of positive genes were annotated as hydrothermal carbonisation (HTC) in leaves or roots and involved in the oxidation-reduction pathway, such as OG06179, OG06849, and OG05048. OG09458 belongs to ubiquinone oxidoreductase, also functions in HTC in the leaf. OG05094 is annotated as 2-aminoethanethiol dioxygenaselike protein. In addition, there were three developmentrelated genes found to have been subjected to positive selection, a thiamin pyrophosphokinase (TPK) encoding gene (OG07167) and two Ca2+-dependent phospholipidbinding protein encoding genes (OG06647 and OG05019).

OG07232 and OG07554 were E3 ubiquitin ligases which respond to drought stress in maize [59] and involved in rice antiviral defense in the early stages of rice dwarf virus infection [63]. We identified two F-box/kelch repeat proteins OG09141 and OG08464 that are positively selected. OG06038 and OG03732 were annotated as Calvin cycle protein and early nodulin 16 precursor, respectively, which also involved in plant response to stresses and rapid environmental changes [64-67]. However, further experiments and field observations are needed to discuss whether/how the functions of those positively selected genes were promoted during domestication.

\section{Conclusions}

Previous studies have shown that domestication-related traits are often controlled by few genetic loci. However, the selective sweeps in eggplants remain unknown. We identified 44,073-58,677 unigenes for six wild and cultivated eggplants; the average number of unigenes for the three $S$. melongena species (55,500 genes) and the N50 values (1,368 1,576 bp) are both higher than that for the recent transcriptomic study on eggplant [33]. The phylogenetic tree revealed that the three cultivated eggplants in section Melongena (HZHQ, LYQ, and S58) were grouped into the same clade; S. aethiopicum and S. integrifolium from section Oliganthes were included in the sister clade, close to the cultivated eggplants, whereas the wild species $S$. sisymbriifolium in the Sisymbriifolium clade was in the most distant clade from the domesticated species. Functional analysis of species-specific genes indicate that eggplant has gone through complex molecular and metabolic changes during domestication, evolving its own gene pool with increased new genes as well as rapid adaptation to different environmental conditions. Moreover, relatively few mutations (19 positively selected genes) have 


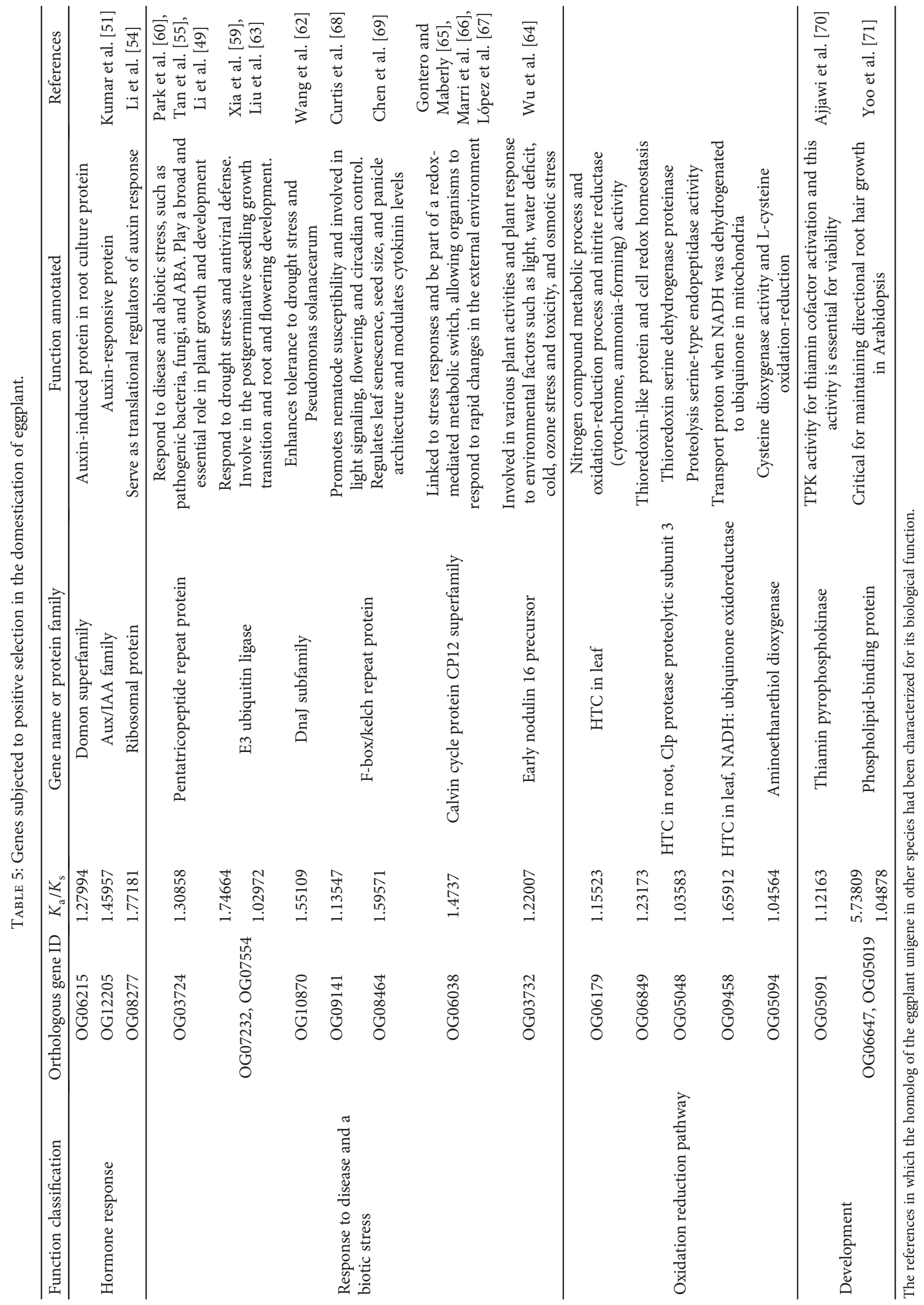


gone through strong selective sweeps during eggplant domestication. Our results provide insights into the selection patterns of the transcriptomes of wild and cultivated eggplants and the understanding of complex domesticatedrelated traits.

\section{Data Availability}

The raw data of RNA-seq experiment is deposited in the Sequence Read Archive (NCBI): SRP127743.

\section{Conflicts of Interest}

The authors declare that they have no competing interests.

\section{Authors' Contributions}

QZW and LMD conceived and designed the experiments, performed the experiments, analyzed the data, prepared the figures and/or tables, authored or reviewed drafts of the paper, and approved the final draft. WHW analyzed the data, authored or reviewed drafts of the paper, and approved the final draft. THH, HJH, and JLW analyzed the data, contributed reagents/materials/analysis tools, authored or reviewed drafts of the paper, and approved the final draft. CLB and $\mathrm{KD}$ conceived and designed the experiments, authored or reviewed drafts of the paper, and approved the final draft.

\section{Acknowledgments}

The present study was supported by the Natural Science Foundation of Zhejiang Province (grant number LQ18C150004), Major Science and Technology Projects of Zhejiang (grant number 2016C02051-2-1), and Local Science and Technology Cooperation Project (grant number LS2017004).

\section{Supplementary Materials}

Table S1: detailed GO annotation of species-specific genes for the three wild eggplant species and two cultivars. Table S2: specific GO terms for the three wild eggplant species and two cultivars. Table S3: overrepresented KEGG pathway enrichment among genes with higher expression based on the interspecific pairwise gene expression comparisons. Table S4: primer sequences for the 16 random selected genes used in qPCR analysis. (Supplementary Materials)

\section{References}

[1] C. Darwin, The variation of animals and plants under domestication, Vol. 2, Cambridge University Press, 2010.

[2] J. F. Doebley, B. S. Gaut, and B. D. Smith, "The molecular genetics of crop domestication," Cell, vol. 127, no. 7, pp. 1309-1321, 2006.

[3] H. G. Parker, A. L. Shearin, and E. A. Ostrander, "Man's best friend becomes biology's best in show: genome analyses in the domestic dog," Annual Review of Genetics, vol. 44, no. 1, pp. 309-336, 2010.
[4] T. Izawa, S. Konishi, A. Shomura, and M. Yano, "DNA changes tell us about rice domestication," Current Opinion in Plant Biology, vol. 12, no. 2, pp. 185-192, 2009.

[5] I. Paran and E. van der Knaap, "Genetic and molecular regulation of fruit and plant domestication traits in tomato and pepper," Journal of Experimental Botany, vol. 58, no. 14, pp. 3841-3852, 2007.

[6] B. L. Gross and K. M. Olsen, "Genetic perspectives on crop domestication," Trends in Plant Science, vol. 15, no. 9, pp. 529-537, 2010.

[7] X. Huang, N. Kurata, X. Wei et al., "A map of rice genome variation reveals the origin of cultivated rice," Nature, vol. 490, no. 7421, pp. 497-501, 2012.

[8] D. Koenig, J. M. Jimenez-Gomez, S. Kimura et al., "Comparative transcriptomics reveals patterns of selection in domesticated and wild tomato," Proceedings of the National Academy of Sciences of the United States of America, vol. 110, no. 28, pp. E2655-E2662, 2013.

[9] R. Swanson-Wagner, R. Briskine, R. Schaefer et al., "Reshaping of the maize transcriptome by domestication," Proceedings of the National Academy of Sciences of the United States of America, vol. 109, no. 29, pp. 11878-11883, 2012.

[10] C. Qin, C. Yu, Y. Shen et al., "Whole-genome sequencing of cultivated and wild peppers provides insights into Capsicum domestication and specialization," Proceedings of the National Academy of Sciences of the United States of America, vol. 111, no. 14, pp. 5135-5140, 2014.

[11] M. C. Daunay, R. N. Lester, C. Gebhardt et al., "Genetic resources of eggplant (Solanum melongena L.) and allied species: a new challenge for molecular geneticists and eggplant breeders," in Advances in Taxonomy and Utilization, V. Solanaceae, Ed., pp. 251-274, Nijmegen University Press, Nijmegen, The Netherlands, 2001.

[12] R. S. Meyer, K. G. Karol, D. P. Little, M. H. Nee, and A. Litt, "Phylogeographic relationships among Asian eggplants and new perspectives on eggplant domestication," Molecular Phylogenetics and Evolution, vol. 63, no. 3, pp. 685-701, 2012.

[13] H. Hirakawa, K. Shirasawa, K. Miyatake et al., "Draft genome sequence of eggplant (Solanum melongena L.): the representative solanum species indigenous to the old world," DNA Research, vol. 21, no. 6, pp. 649-660, 2014.

[14] P. Gramazio, J. Blanca, P. Ziarsolo et al., "Transcriptome analysis and molecular marker discovery in Solanum incanum and S. aethiopicum, two close relatives of the common eggplant (Solanum melongena) with interest for breeding," BMC Genomics, vol. 17, no. 1, p. 300, 2016.

[15] K. R. Ramesh, R. Hemalatha, C. A. Vijayendra, U. Z. S. Arshi, S. B. Dushyant, and K. B. Dinesh, "Transcriptome analysis of Solanum melongena L. (eggplant) fruit to identify putative allergens and their epitopes," Gene, vol. 576, no. 1, pp. 64-71, 2016.

[16] F. Chen, A. J. Mackey, J. K. Vermunt, and D. S. Roos, "Assessing performance of orthology detection strategies applied to eukaryotic genomes," PLoS One, vol. 2, no. 4, article e383, 2007.

[17] J. Li, Y. J. He, L. Zhou et al., “Transcriptome profiling of genes related to light-induced anthocyanin biosynthesis in eggplant (Solanum melongena L.) before purple color becomes evident," BMC Genomics, vol. 19, no. 1, p. 201, 2018.

[18] B. Song, Y. Song, Y. Fu et al., "Draft genome sequence of the Solanum aethiopicum provides insights into disease resistance, 
drought tolerance and the evolution of the genome," BioRxiv Preprint, 2019.

[19] S. Stern, M. F. Agra, and L. Bohs, "Molecular delimitation of clades within New World species of the "spiny solanums" (Solanum subg. Leptostemonum)," Taxon, vol. 60, no. 5, pp. 1429-1441, 2011.

[20] M. G. Grabherr, B. J. Haas, M. Yassour et al., "Full-length transcriptome assembly from RNA-Seq data without a reference genome," Nature Biotechnology, vol. 29, no. 7, pp. 644652, 2011.

[21] M. D. Young, M. J. Wakefield, G. K. Smyth, and A. Oshlack, "Gene ontology analysis for RNA-seq: accounting for selection bias," Genome Biology, vol. 11, no. 2, article R14, 2010.

[22] M. Kanehisa, M. Araki, S. Goto et al., "KEGG for linking genomes to life and the environment," Nucleic Acids Research, vol. 36, Supplement_1, pp. D480-D484, 2007.

[23] X. Mao, T. Cai, J. G. Olyarchuk, and L. Wei, "Automated genome annotation and pathway identification using the KEGG Orthology (KO) as a controlled vocabulary," Bioinformatics, vol. 21, no. 19, pp. 3787-3793, 2005.

[24] B. Li and C. N. Dewey, "RSEM: accurate transcript quantification from RNA-Seq data with or without a reference genome," BMC Bioinformatics, vol. 12, no. 1, p. 323, 2011.

[25] A. Mortazavi, B. A. Williams, K. McCue, L. Schaeffer, and B. Wold, "Mapping and quantifying mammalian transcriptomes by RNA-Seq," Nature Methods, vol. 5, no. 7, pp. 621$628,2008$.

[26] K. J. Livak and T. D. Schmittgen, "Analysis of relative gene expression data using real-time quantitative PCR and the $2^{-\Delta \Delta C}$ method," Methods, vol. 25, no. 4, pp. 402-408, 2001.

[27] M. Zhang and H. W. Leong, "Bidirectional best hit $r$-window gene clusters," BMC Bioinformatics, vol. 11, article S63, Supplement 1, 2010.

[28] A. Doron-Faigenboim, A. Stern, I. Mayrose, E. Bacharach, and T. Pupko, "Selecton: a server for detecting evolutionary forces at a single amino-acid site," Bioinformatics, vol. 21, no. 9, pp. 2101-2103, 2005.

[29] A. Stàgel, E. Portis, L. Toppino, G. Rotino, and S. Lanteri, "Gene-based microsatellite development for mapping and phylogeny studies in eggplant," BMC Genomics, vol. 9, no. 1, p. 357, 2008.

[30] K. Demir, M. Bakır, G. Sarıkamış, and S. Acunalp, "Genetic diversity of eggplant (Solanum melongena) germplasm from Turkey assessed by SSR and RAPD markers," Genetics and Molecular Research, vol. 9, no. 3, pp. 1568-1576, 2010.

[31] H. Li, H. Chen, T. Zhuang, and J. Chen, "Analysis of genetic variation in eggplant and related Solanum species using sequence-related amplified polymorphism markers," Scientia Horticulturae, vol. 125, no. 1, pp. 19-24, 2010.

[32] Y. Tümbilen, A. Frary, M. C. Daunay, and S. Doğanlar, "Application of EST-SSRs to examine genetic diversity in eggplant and its close relatives," Turkish Journal of Biology, vol. 35, no. 2, p. 125, 2011.

[33] X. Yang, Y. F. Cheng, C. Deng et al., "Comparative transcriptome analysis of eggplant (Solanum melongena L.) and turkey berry (Solanum torvum Sw.): phylogenomics and disease resistance analysis," BMC Genomics, vol. 15, no. 1, p. 412, 2014.

[34] C. Trapnell, L. Pachter, and S. L. Salzberg, "TopHat: discovering splice junctions with RNA-Seq," Bioinformatics, vol. 25, no. 9, pp. 1105-1111, 2009.
[35] L. Li, C. J. Stoeckert Jr., and D. S. Roos, "OrthoMCL: identification of ortholog groups for eukaryotic genomes," Genome Research, vol. 13, no. 9, pp. 2178-2189, 2003.

[36] X. Xu, S. Pan, S. Cheng et al., "Genome sequence and analysis of the tuber crop potato," Nature, vol. 475, no. 7355, pp. 189195, 2011.

[37] The Tomato Genome Consortium, "The tomato genome sequence provides insights into fleshy fruit evolution," Nature, vol. 485, no. 7400, pp. 635-641, 2012.

[38] Y. Wang, A. Diehl, F. Wu et al., "Sequencing and comparative analysis of a conserved syntenic segment in the Solanaceae," Genetics, vol. 180, no. 1, pp. 391-408, 2008.

[39] G. Ano, Y. Hebert, P. Prior, and C. M. Messiaen, “A new source of resistance to bacterial wilt of eggplants obtained from a cross: Solanum aethiopicum $\mathrm{L} \times$ Solanum melongena $\mathrm{L}$," Agronomie, vol. 11, no. 7, pp. 555-560, 1991.

[40] C. Collonnier, K. Mulya, I. Fock et al., "Source of resistance against Ralstonia solanacearum in fertile somatic hybrids of eggplant (Solanum melongena L.) with Solanum aethiopicum L," Plant Science, vol. 160, no. 2, pp. 301-313, 2001.

[41] C. Collonnier, I. Fock, M. C. Daunay et al., "Somatic hybrids between Solanum melongena and S. sisymbrifolium, as a useful source of resistance against bacterial and fungal wilts," Plant Science, vol. 164, no. 5, pp. 849-861, 2003.

[42] L. Toppino, G. Valè, and G. L. Rotino, "Inheritance of Fusarium wilt resistance introgressed from Solanum aethiopicum Gilo and Aculeatum groups into cultivated eggplant (S. melongena) and development of associated PCR-based markers," Molecular Breeding, vol. 22, no. 2, pp. 237-250, 2008.

[43] C. Kaur, S. Nagal, J. Nishad, R. Kumar, and Sarika, "Evaluating eggplant (Solanum melongena L.) genotypes for bioactive properties: a chemometric approach," Food Research International, vol. 60, no. 6, pp. 205-211, 2014.

[44] S. I. Wright, I. V. Bi, S. G. Schroeder et al., "The effects of artificial selection on the maize genome," Science, vol. 308, no. 5726, pp. 1310-1314, 2005.

[45] G. Jia, X. Huang, H. Zhi et al., "A haplotype map of genomic variations and genome-wide association studies of agronomic traits in foxtail millet (Setaria italica)," Nature Genetics, vol. 45, no. 8, pp. 957-961, 2013.

[46] J. Qi, X. Liu, D. Shen et al., "A genomic variation map provides insights into the genetic basis of cucumber domestication and diversity," Nature Genetics, vol. 45, no. 12, pp. 15101515, 2013.

[47] M. B. Hufford, X. Xu, J. van Heerwaarden et al., "Comparative population genomics of maize domestication and improvement," Nature Genetics, vol. 44, no. 7, pp. 808-811, 2012.

[48] Z. Zhang and J. Yu, "Evaluation of six methods for estimating synonymous and nonsynonymous substitution rates," Genomics, Proteomics \& Bioinformatics, vol. 4, no. 3, pp. 173181, 2006.

[49] X. J. Li, Y. F. Zhang, M. Hou et al., "Small kernel 1 encodes a pentatricopeptide repeat protein required for mitochondrial nad7 transcript editing and seed development in maize (Zea mays) and rice (Oryza sativa)," The Plant Journal, vol. 79, no. 5, pp. 797-809, 2014.

[50] M. de Jong, M. Wolters-Arts, B. C. J. Schimmel et al., "Solanum lycopersicum AUXIN RESPONSE FACTOR 9 regulates cell division activity during early tomato fruit development," Journal of Experimental Botany, vol. 66, no. 11, pp. 3405-3416, 2015. 
[51] R. Kumar, A. K. Tyagi, and A. K. Sharma, "Genome-wide analysis of auxin response factor (ARF) gene family from tomato and analysis of their role in flower and fruit development," Molecular Genetics and Genomics, vol. 285, no. 3, pp. 245-260, 2011.

[52] R. Kumar, P. Agarwal, A. Pareek, A. K. Tyagi, and A. K. Sharma, "Genomic survey, gene expression, and interaction analysis suggest diverse roles of ARF and Aux/IAA proteins in Solanaceae," Plant Molecular Biology Reporter, vol. 33, no. 5, pp. 1552-1572, 2015.

[53] L. Su, C. Audran, M. Bouzayen, J. P. Roustan, and C. Chervin, "The Aux/IAA, Sl-IAA17 regulates quality parameters over tomato fruit development," Plant Signaling \& Behavior, vol. 10, no. 11, article e1071001, 2015.

[54] R. Li, R. Sun, G. R. Hicks, and N. V. Raikhel, "Arabidopsis ribosomal proteins control vacuole trafficking and developmental programs through the regulation of lipid metabolism," Proceedings of the National Academy of Sciences of the United States of America, vol. 112, no. 1, pp. E89-E98, 2015.

[55] J. Tan, Z. Tan, F. Wu et al., "A novel chloroplast-localized pentatricopeptide repeat protein involved in splicing affects chloroplast development and abiotic stress response in rice," Molecular Plant, vol. 7, no. 8, pp. 1329-1349, 2014.

[56] G. H. He, J. Y. Xu, Y. X. Wang et al., "Drought-responsive WRKY transcription factor genes TaWRKY1 and TaWRKY33 from wheat confer drought and/or heat resistance in Arabidopsis," BMC Plant Biology, vol. 16, no. 1, p. 116, 2016.

[57] Y. Zhang, H. Yu, X. Yang et al., "CsWRKY46, a WRKY transcription factor from cucumber, confers cold resistance in transgenic-plant by regulating a set of cold-stress responsive genes in an ABA-dependent manner," Plant Physiology and Biochemistry, vol. 108, pp. 478-487, 2016.

[58] J. Wang, F. Tao, F. An et al., "Wheat transcription factor TaWRKY70 is positively involved in high-temperature seedling-plant resistance to Puccinia striiformis f. sp. tritici," Molecular Plant Pathology, vol. 18, no. 5, pp. 649-661, 2017.

[59] Z. Xia, Q. Liu, J. Wu, and J. Ding, "ZmRFP1, the putative ortholog of SDIR1, encodes a RING-H2 E3 ubiquitin ligase and responds to drought stress in an ABA-dependent manner in maize," Gene, vol. 495, no. 2, pp. 146-153, 2012.

[60] Y. J. Park, H. J. Lee, K. J. Kwak, K. Lee, S. W. Hong, and H. Kang, "MicroRNA400-guided cleavage of pentatricopeptide repeat protein mRNAs renders Arabidopsis thaliana more susceptible to pathogenic bacteria and fungi," Plant and Cell Physiology, vol. 55, no. 9, pp. 1660-1668, 2014.

[61] C. Colas des Francs-Small, A. Falcon de Longevialle, Y. Li et al., "The pentatricopeptide repeat proteins TANG2 and ORGANELLE TRANSCRIPT PROCESSING439 are involved in the splicing of the multipartite nad5 transcript encoding a subunit of mitochondrial complex I," Plant Physiology, vol. 165, no. 4, pp. 1409-1416, 2014.

[62] G. Wang, G. Cai, F. Kong, Y. Deng, N. Ma, and Q. Meng, "Overexpression of tomato chloroplast-targeted DnaJ protein enhances tolerance to drought stress and resistance to Pseudomonas solanacearum in transgenic tobacco," Plant Physiology and Biochemistry, vol. 82, no. 3, pp. 95-104, 2014.

[63] L. Liu, L. Jin, X. Huang et al., "OsRFPH2-10, a ring-H2 finger E3 ubiquitin ligase, is involved in rice antiviral defense in the early stages of rice dwarf virus infection," Molecular Plant, vol. 7, no. 6, pp. 1057-1060, 2014.

[64] H. Wu, Y. Shen, Y. Hu, S. Tan, and Z. Lin, "A phytocyaninrelated early nodulin-like gene, $B c B C P 1$, cloned from Boea crassifolia enhances osmotic tolerance in transgenic tobacco," Journal of Plant Physiology, vol. 168, no. 9, pp. 935-943, 2011.

[65] B. Gontero and S. C. Maberly, "An intrinsically disordered protein, CP12: jack of all trades and master of the Calvin cycle," Biochemical Society Transactions, vol. 40, no. 5, pp. 995-999, 2012.

[66] L. Marri, G. Thieulin-Pardo, R. Lebrun et al., "CP12-mediated protection of Calvin-Benson cycle enzymes from oxidative stress," Biochimie, vol. 97, no. 1, pp. 228-237, 2014.

[67] P. E. López-Calcagno, T. P. Howard, and C. A. Raines, "The CP12 protein family: a thioredoxin-mediated metabolic switch?" Frontiers in Plant Science, vol. 5, no. 3, p. 9, 2014.

[68] R. H. C. Curtis, Pankaj, S. J. Powers, J. Napier, and M. C. Matthes, "The Arabidopsis F-box/Kelch-repeat protein At2g44130 is upregulated in giant cells and promotes nematode susceptibility," Molecular Plant-Microbe Interactions, vol. 26, no. 1, pp. 36-43, 2013.

[69] Y. Chen, Y. Xu, W. Luo et al., "The F-Box protein OsFBK12 targets OsSAMS1 for degradation and affects pleiotropic phenotypes, including leaf senescence, in rice," Plant Physiology, vol. 163, no. 4, pp. 1673-1685, 2013.

[70] I. Ajjawi, M. A. Rodriguez Milla, J. Cushman, and D. K. Shintani, "Thiamin pyrophosphokinase is required for thiamin cofactor activation in Arabidopsis," Plant Molecular Biology, vol. 65, no. 1-2, pp. 151-162, 2007.

[71] C. M. Yoo, J. Wen, C. M. Motes, J. A. Sparks, and E. B. Blancaflor, "A class I ADP-ribosylation factor GTPaseactivating protein is critical for maintaining directional root hair growth in Arabidopsis," Plant Physiology, vol. 147, no. 4, pp. 1659-1674, 2008. 


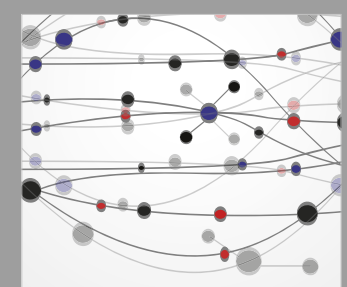

The Scientific World Journal
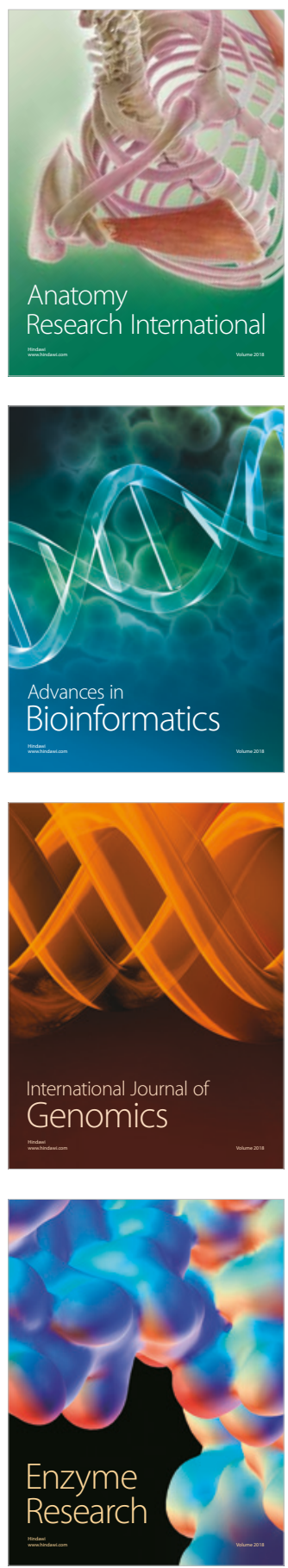
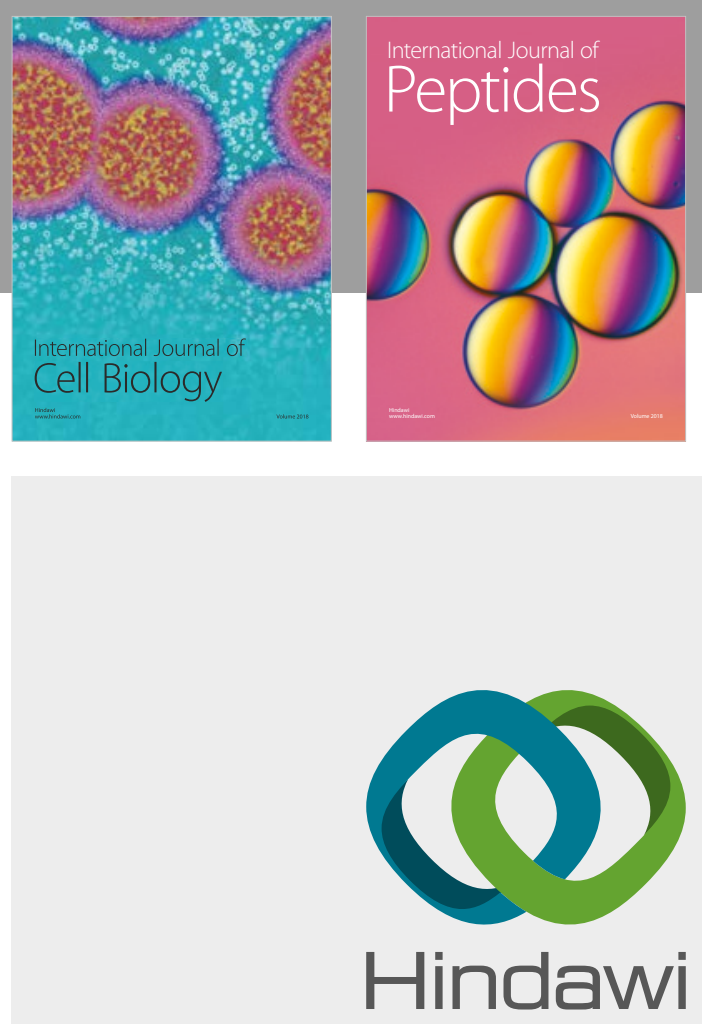

Submit your manuscripts at

www.hindawi.com
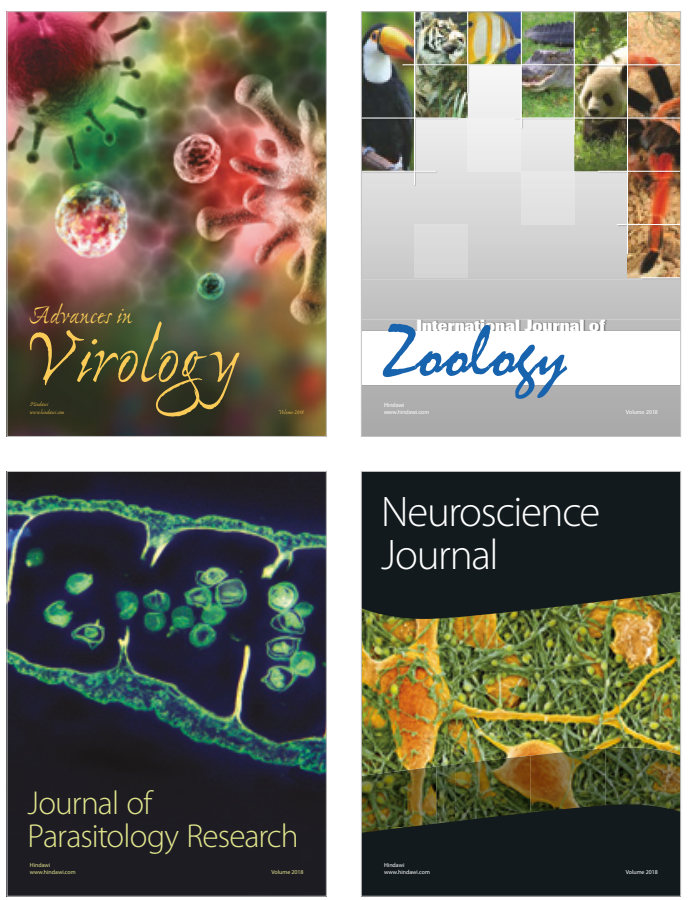
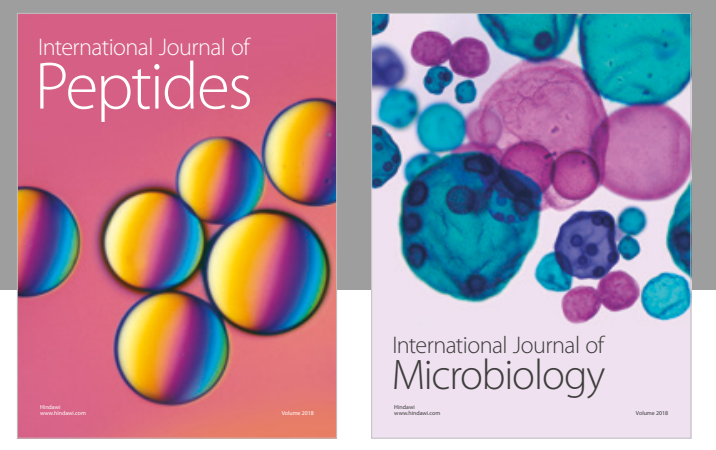

nternational Journal of Microbiology
Journal of
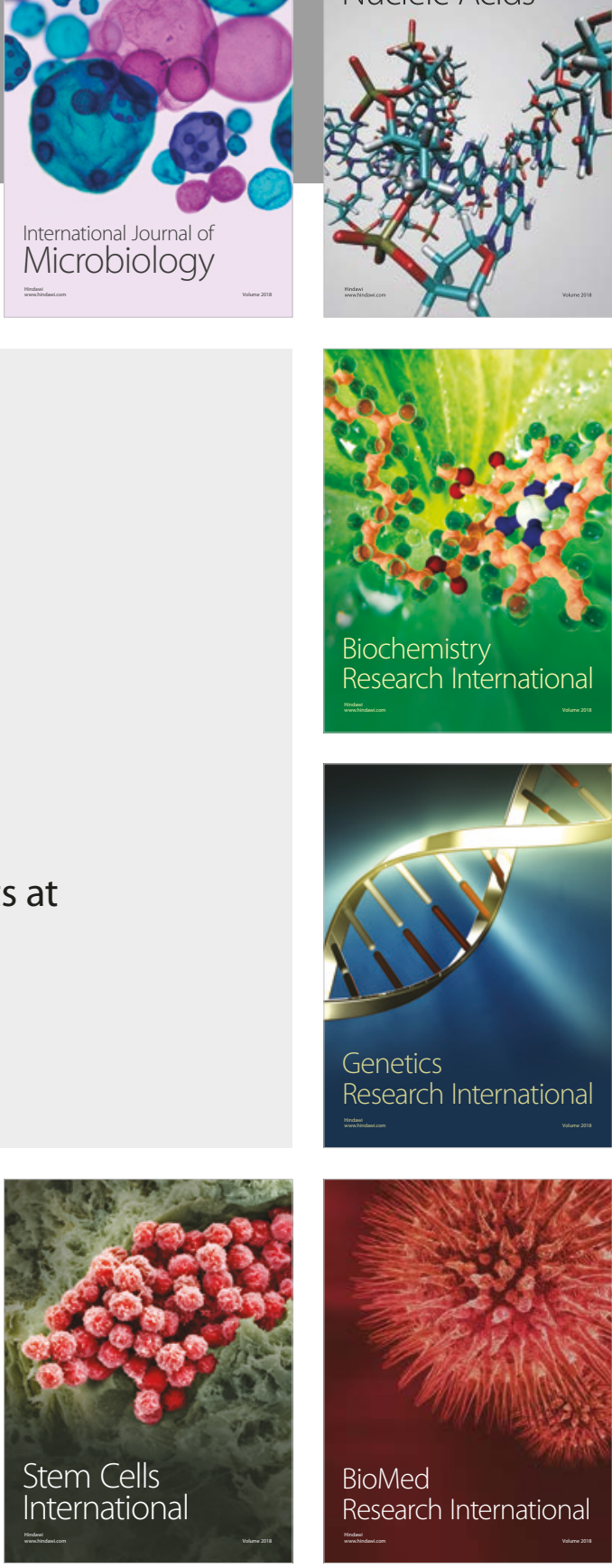
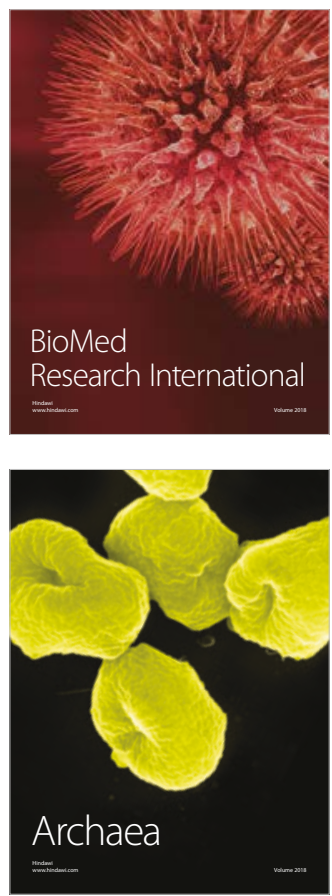